\title{
PRONAÇÃO DO IDOSO NA COVID-19: CONSIDERAÇÕES DA ENFERMAGEM GERONTOLÓGICA
}

\author{
Graziele Ribeiro Bitencourt' \\ ORCID: 0000-0002-9130-9307 \\ Antonia Rios Almeida" \\ ORCID: 0000-0003-1615-5042
}

Bruna Luisa Melo de Aquino Lemos Corrêa"I ORCID: 0000-0002-6777-572X

Greiciane da Silva Rochaiv ORCID: 0000-0002-1636-7179

Roseni dos Santos Souzav ORCID: 0000-0002-0061-725X

Vanessa Macedo Couto ${ }^{\text {VI }}$ ORCID: 0000-003-387-6449

Rosimere Ferreira Santana ${ }^{\text {vII }}$ ORCID: 0000-0002-4593-3715

'Universidade Federal do Rio de Janeiro. Campus Macaé. Macaé, Rio de Janeiro, Brasil.

"Instituto Nacional do Cancer. Rio de Janeiro, Rio de Janeiro, Brasil.

"'Hospital Santa Juliana. Rio Branco, Acre, Brasil.

"Universidade Federal do Acre. Rio Branco, Acre, Brasil.

${ }^{v}$ Hospital Municipal Ronaldo Gazolla. Rio de Janeiro, Rio de Janeiro, Brasil.

vUniversidade Federal Fluminense. Hospital Universitário Antônio Pedro. Niterói, Rio de Janeiro, Brasil.

"I"Universidade Federal Fluminense. Niterói, Rio de Janeiro, Brasil.

Autor Correspondente: Graziele Ribeiro Bitencourt E-mail: grazielebitencourt@macae.ufrj.br

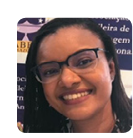

Como citar:

Bitencourt GR, Almeida AR, Corrêa BLMAL, Rocha GS, Souza RS, Couto VM, Santana RF. Pronação do Idoso na Covid-19: Considerações de Enfermagem Gerontológica. In: Santana RF (Org.). Enfermagem gerontológica no cuidado do idoso em tempos da COVID 19. Brasilia, DF: Editora ABen; 2021. 171 p. (Serie Enfermagem e Pandemias, 5). https://doi.org/10.51234/aben.21.e05.c15

\section{INTRODUÇÃO}

A pandemia COVID-19 desafia os sistemas de saúde com um número elevado de pacientes que precisam de cuidados intensivos. A idade é relacionada ao aumento de casos e piora do prognóstico, seja pela presença de comorbidades ou por alterações pulmonares do processo de envelhecimento.

Os casos mais graves cursam com insuficiência respiratória devido à, principalmente, síndrome respiratória aguda grave e consequente intubação de difícil controle. A posição prona é uma das estratégias paralelas a essa intubação para melhora da expansibilidade pulmonar e é conhecida por reduzir a mortalidade nos casos moderados e graves ${ }^{(1)}$.

No paciente adulto, há indícios de melhora no quadro respiratório. Estudos apontam evidências positivas na relação perfusão/ventilação e redução da hipoxemia pela aeração mais homogênea do pulmão e do gradiente de pressão transpulmonar ventral-dorsal; redução do shunt pulmonar; recrutamento dos segmentos pulmonares posteriores devido à reversão da atelectasia; e drenagem de secreção das vias aéreas ${ }^{(2)}$.

Entretanto, há risco de complicações que podem ser fatais, como a extubação acidental e avulsão de cateter central. Outras complicações menos graves podem ser observadas como lesões por pressão (LPP) em região facial, tórax e joelho, necrose mamária; edema facial, de membros e tórax; lesão de plexo braquial; intolerância à dieta; extubação acidental; seletividade; deslocamento e obstrução do tubo endotraqueal; e remoção e dificuldade de fluxo no cateter de hemodiálise e outros cateteres, além de remoção de sondas enterais e vesicais( ${ }^{(3)}$.

Associada ao paciente idoso, há especificidades que podem comprometer a manobra e aumentar o risco da posição, como diminuição da amplitude de movimentos, vasculites, osteoporose, comprometimento cardíaco e alterações digestivas com o aumento do risco de broncoaspiração. 
Há ainda a maior chance de insucesso da manobra pelos comprometimentos pulmonares preexistentes no idoso, como diminuição da expansibilidade pulmonar ${ }^{(4)}$.

Faz-se necessária, portanto, a expertise da enfermagem gerontológica para dar atenção às particularidades no idoso e aos risco inerentes relacionado a posição prona, desde sua indicação, cuidados pré, durante e após a manobra, além da prevenção de complicações.

Nesse sentido, questiona-se: Como a pronação pode ser estabelecida como estratégia de cuidado ao paciente idoso no contexto da COVID-19?

\section{OBJETIVO}

Descrever as considerações da enfermagem gerontológica no processo de pronação do paciente idoso com COVID-19.

\section{MÉTODO}

Trata-se de um estudo descritivo, do tipo análise reflexiva da literatura, baseado em artigos científicos e normas técnicas publicadas no ano de 2020.

\section{RESULTADOS}

No contexto da COVID-19 precisa-se refletir como a posição prona pode ser considerada no cuidado ao idoso, conforme a Figura 1.

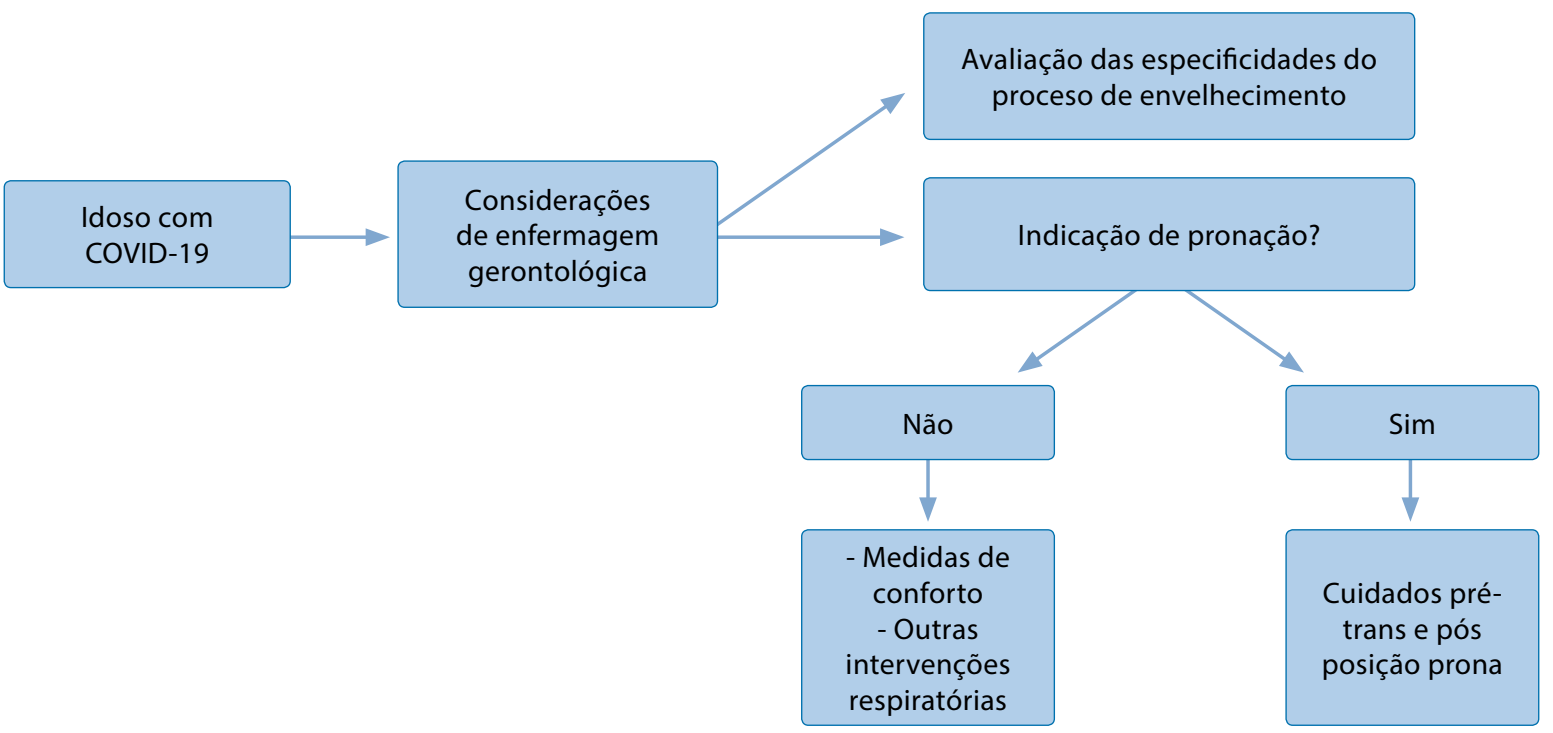

Figura 1 - Posição prona no paciente idoso no contexto da COVID-19

O idoso com COVID-19 requer avaliação de aspectos gerontológicos. Neste sentido, há necessidade de consideração das especificidades do processo de envelhecimento para indicação ou não da posição prona enquanto intervenção de enfermagem. As principais situações de risco deste posicionamento necessitam 
de análise na seleção e, se indicado, o monitoramento, o qual inclui cuidados pré-manobra, incluindo sua recomendação assertiva, além da avaliação após finalização.

\section{Aspectos Legais}

A resolução Cofen n ${ }^{\circ}$ 639, de 06 de maio de 2020 dispõe sobre as competências do Enfermeiro no cuidado aos pacientes em ventilação mecânica no ambiente extra e intra-hospitalar. A pronação se enquadra. No Art.3, o parágrafo $V$ estabelece que cabe ao enfermeiro:

participar da decisão, da realização e/ou prescrição na Equipe de Enfermagem dos procedimentos relacionados à pronação de pacientes sob ventilação mecânica e aplicação dos cuidados relacionados a prevenção dos incidentes associados.

Neste sentido, também cabe a enfermagem compreender as características de cada paciente e considerar a indicação, cuidados pré, durante e após a manobra, além da identificação precoce das principais complicações.

\section{Indicações da posição prona}

Em virtude das características clínicas de necessidade ao suporte ventilatório, em decorrência do quadro hipoxêmico grave, considera-se a posição prona no leito, uma medida colaborativa na terapêutica de pacientes infectados pelo COVI-19. Indica-se a posição prona em pacientes que possam cooperar com o procedimento sem problemas preditores de vias aéreas e com independência de mobilidade ${ }^{(1)}$.

A pronação se faz necessária na presença de $\mathrm{PO} 2 / \mathrm{FiO} 2<150$. Esse tipo de ventilação tem boa eficácia nos pacientes com quadro da síndrome do desconforto respiratório agudo (SDRA) e em pacientes com quadro similar da síndrome relacionado ao COVID-19. A eficiência dessa ventilação se pauta da distribuição da tensão pulmonar com melhora da ventilação/perfusão com aumento do volume expiratório final, elasticidade da parece torácica, melhora do volume corrente e com a diminuição do shunt alveolar ${ }^{(5)}$.

Além disso, precisa ser considerado o início do curso da doença (idealmente inferior a 48 horas) após 1224 horas de ventilação mecânica. Há estudos em adultos que apontam melhores resultados obtidos usando volumes correntes de $6 \mathrm{ml} / \mathrm{kg}$ de peso corporal previsto e considerar o uso de bloqueadores neuromusculares se houver evidência de dessincronia ventilatória. Deve ser considerada ainda a relação de pressão parcial de oxigênio arterial - $\mathrm{PaO} 2$ e fração inspirada de oxigênio - FiO2 (Pa02/FiO2) menor a 150 mm Hg ${ }^{(6)}$.

Porém, vale-se considerar que se faz necessário uma avaliação clínica rigorosa em pacientes idosos e suas comorbidades, visto que eles podem não tolerar a posição pelo seu desconforto, o que pode levar ao quadro de ansiedade e piora do quadro. É preciso o envolvimento profissional multidisciplinar para evitar iatrogenias e maiores complicações durante o tratamento(2).

Por outro lado, não há estudos claros sobre sua eficácia na clientela idosa que assegurem a sua indicação. Sabe-se que o idoso apresenta variações pulmonares como diminuição da capacidade máxima de respiração, do fluxo respiratório forçado e da pressão parcial de oxigênio, aumento da capacidade residual funcional ${ }^{(4)}$ que podem ser agravadas na COVID-19. Além disso, há comprometimento osteoarticular e muscular que também precisa ser considerado para a indicação e análise da tolerância do tempo de posicionamento pelo paciente idoso.

\section{Cuidados pré-manobra}

Para a realização da posição prona é necessário uma equipe multidisciplinar discuta sobre os riscos e benefícios potenciais da ventilação prona, além da análise sobre as contraindicações do procedimento, sendo 
as principais, instabilidade na coluna vertebral presente na maioria dos idosos, tórax aberto após cirurgia / trauma cardíaco, além de período inferior a 24 horas de cirurgia cardíaca. É necessário ainda de treinamento dessa equipe para a minimização de riscos da prática assistencial( ${ }^{(6)}$.

A fim de melhorar a qualidade no atendimento sugere-se que seja disponibilizado de forma viável, uma padronização nos horários para o manejo adequado e eficiente ao paciente em posição prona. A sequência dos dados pode ser obtida através de uma padronização da técnica através da utilização de um instrumento que a norteie tais como: checklist, protocolo, diretrizes, instruções de trabalho acerca dos cuidados de enfermagem, que sejam de acordo com as características da instituição que as implanta. Dessa forma, ficará estabelecido que o líder deverá acionar e/ou gerenciar o comando para a equipe de forma clara e concisa. Estudos sugerem que a incidência de eventos adversos é significativamente reduzida na presença de padrões e uma equipe treinada, tornando a manobra segura ${ }^{(7)}$.

Além disso, é necessário garantir um número adequado de profissionais disponíveis para facilitar o procedimento seguro. Em média, é recomendado o mínimo de 5, sendo 2 em cada lateral e um na cabeça responsável pela estabilização do tudo orotraqueal. Todos os dispositivos invasivos precisam ser certificados quanto a segurança no posicionamento e estabilidade, principalmente o tubo orotraqueal, dreno torácico, sonda para dietoterapia e cateter vesical, visando reduzir o risco de desposicionamento acidental. Todas infusões e monitoramento não essenciais precisam ser pausados $O$ paciente deve estar recebendo sedação e analgesia adequadas e a utilização de escalas minutos antes da realização do procedimento é fortemente recomentada ${ }^{(3)}$.

Deve ser realizada aspiração das vias aéreas e da orofaringe antes do procedimento. Faz-se necessária a garantia das configurações adequadas do ventilador mecânico e a pré-oxigenação com $100 \%$ de oxigênio. Entretanto, nos casos de doença pulmonar obstrutiva crônica(DPOC) presente em parte dos idosos, essa prática precisa ser avaliada com cautela. É necessário ainda realizar gasometria arterial pré-pronação e documentar os resultados e a monitorização cardiovascular. Caso esteja não esteja estável, o procedimento não será realizado(6).

Ainda anterior ao procedimento, é necessário o registro da integridade da pele da pele do paciente e a limpeza, lubrificação, e proteção com gaze ou fechamento com fita adesiva para evitar ressecamento e ulceração ${ }^{(2,6)}$. No processo de envelhecimento, esse ressecamento ocular e comprometimento cutâneo são ainda mais arriscados, trazendo a necessidade ainda maior de atenção.

\section{Cuidados durante a manobra}

O paciente em pronação, geralmente, está internado em setor de cuidados intensivos e dispõe de vários dispositivos invasivos e não invasivos tais como: tubo orotraqueal, sonda nasoentérica, sonda vesical, cateter de hemodiálise, cateter venoso central, monitorização cardíaca, oximetria de pulso, dentre outros. Em conjunto, esses dispositivos devem ser monitorados de forma ainda mais rigorosa para preservar a integridade do paciente durante a manobra ${ }^{(6)}$.

Os principais cuidados dispensados pela equipe durante a execução devem ser: dispor de uma equipe posicionada, preferencialmente, na cabeceira, laterais do tórax, membros inferiores; posicionar eletrodos e dômus de pressão arterial invasiva nos membros superiores; manter os cabos de monitorização e oximetria alinhados; retirar a dieta e fechar a sonda nasoentérica antes do procedimento que, embora não haja um consenso, precisa ser realizado de 10 a 60 minutos antes; desconectar o extensor de aspiração; posicionar as sondas e drenos devem entre as pernas e ou braços dos pacientes; posicionar planamente a cabeceira e alinhar membros; posicionar coxins na pelve e tórax; interromper infusões e desconectar equipos; formar o envelope de lençol; realizar o giro ao lado oposto do ventilador mecânico ${ }^{(7)}$.

No que tange aos cuidados na manobra referente ao paciente idoso vale salientar as alterações osteoarticulares durante o procedimento, visando dar mais segurança durante o procedimento. A fragilidade da 
integridade da pele pode ocasionar lesões cutâneas e o tempo de permanência na posição prona deve ser cronometrado. A monitorização hemodinâmica precisa ser rigorosa durante esse processo e, em caso de gravidade, pode ser necessário o retorno a posição supina.

\section{Complicações e cuidados após a manobra}

No paciente idoso, a posição prona representa como evento adverso principal a LPP, devido ao aumento de pressão nas proeminências ósseas como ombros, narina, bochechas, região frontal, mandíbula, esterno e cristas ilíacas. A LPP pode prejudicar a recuperação e, se não prevenidas, causar infecções graves como a sepse e a consequente em internação prolongada, além do aumento de mortalidade. Os idosos internados em terapia intensiva tem $66 \%$ de risco no desenvolvimento de LPP. Por isso trata-se de um grupo de risco de maior prevalência de complicações de lesões de pele na indicação de prona ${ }^{(8)}$.

Além disso, o idoso pode apresentar nesta posição uma piora rápida e súbita em seu quadro clínico de insuficiência respiratória. Há ainda o risco de edema facial, instabilidade hemodinâmica transitória, abrasões de córnea, obstrução e infecção no tubo orotraqueal no caso de desconexão acidental. Outros problemas podem estar presentes como flexão de cateteres, edema ao redor dos olhos, refluxo gastresofágico e sialorreia. Portanto, essa clientela deve estar sob constante vigilância e seguir sob a assistência dos cuidados de enfermagem no controle das vias aéreas e detecção de sinais precoces de deterioração(7).

Há também a necessidade de avaliação a cada 4 ou 6 horas dos índices de trocas gasosas. Para isto, o enfermeiro deve realizar a gasometria, a fim de avaliar a resposta ao tratamento, pelo menos uma hora, após a manobra. Não havendo indícios de melhora, é recomendável o retorno do paciente à posição supina. É recomendada ainda a avaliação contínua da Saturação de oxigênio em sangue arterial (SpO2) e da saturação de oxigênio ( $\mathrm{SaO} 2$ ) em níveis de 92 a 96\%, e sem desconforto respiratório(9).

A orientação preconiza que deve ser interrompida a pronação nos seguintes casos: extubação não programada, obstrução do tubo endotraqueal, hemoptise, saturação periférica de oxigênio ( $\mathrm{SpO} 2<85 \%$ ou $\mathrm{PaO} 2$ $<55 \mathrm{mmHg}$ ) por mais de cinco minutos, parada cardiorrespiratória, frequência cardíaca inferior à 30bpm, pressão arterial sistólica inferior à $60 \mathrm{mmHg}$ por mais de cinco minutos. Há contraindicações nos idosos com instabilidade hemodinâmica, com pressão intra-abdominal alta e com lesões no peito, dentre outros ${ }^{(6)}$.

Neste contexto, os cuidados devem ser realizados de forma imediata e contínua. A enfermagem como parte da equipe tem papel fundamental visando sempre a prevenção e minimização dos eventos adversos. Dentre os cuidados pós manobra podem ser descritas a verificação de posicionamento e da pressão do balonete no tubo orotraqueal para evitar lesões na comissura labial e extubação acidental. Além disso, é recomendado o posicionamento em trendelemburg reverso, mudança lateralizada da cabeça/pescoço durante a dieta infusão de enteral para reduzir broncoaspiração e edema de face ${ }^{(7)}$.

A posição de nadador deve ser trocada a cada 2 horas, de modo que um membro fletido para cima e outro estendido para baixo, com rosto virado para o braço fletido. Essa intervenção pode ser realizada até de 4/4 horas, pois neste cenário de pandemia necessita-se de um tempo maior para paramentação de equipamento de proteção individual. Indica-se que dois profissionais executem essa mudança no posicionamento para evitar lesão do plexo braquial do paciente. A utilização de placas de barreiras cutâneas é recomendada como hidrocoloide e/ou filme para regiões ósseas de face, ombros, joelhos e outras proeminências, bem como a colocação de travesseiros e coxins em região de tórax, face e quadril(2).

Desta forma, as principais ações pós-manobra de pronação em ventilação mecânica no idoso são: confirmar a posição do tubo orotraqueal e de traqueostomia; posicionar coxins na região de face, para prevenção de lesões; conectar o sistema de aspiração e verificar se existe dobramento e/ou obstrução de vias áreas (tubo orotraqueal e de traqueostomia); posicionar drenos e cateteres, abrir clamps; reposicionar eletrodos e calibração da pressão arterial invasiva; adicionar coxins em outras regiões para prevenção de lesões; alinhar a posição de nadador; posicionar quadril em leve rotação e fletir levemente os membros inferiores, se viável; 
instalar dieta enteral após 1hora da manobra em baixa vazão; reiniciar infusão das drogas; registrar horário de pronação e monitorar horários subsequentes; mudar o decúbito de cabeça a cada duas horas; observar edema facial; e não realizar RX de tórax, mas sim a ecografia, caso necessário; reavaliar posição de prona após $6 \mathrm{~h}$, com coleta de gasometria; se responsivo a prona manter entre 16 a 20 horas $^{(10)}$.

\section{CONSIDERAÇÕES FINAIS}

A pronação é descrita como uma intervenção de enfermagem possível na melhora da expansão pulmonar no contexto da COVID-19. Entretanto, sua indicação para o idoso precisa de avaliação específica considerando as especificidades do processo de envelhecimento. Nesse caso, faz-se necessário cuidados pré, pós e durante a manobra, com vistas a redução e identificação precoces de complicações.

\section{AGRADECIMENTO}

\section{Ao Departamento Científico de Enfermagem Gerontológica da ABEn Nacional.}

\section{REFERÊNCIAS}

1. Elharrar X, Trigui Y, Dols AM, Touchon F, Martinez S, Prud'homme E, et al. Use of prone positioning in nonintubated patients with COVID-19 and hypoxemic acute respiratory failure. JAMA. 2020;323(22):2336-8. https://doi.org/10.1001/ jama.2020.8255

2. Jiang LG, LeBaron J, Bodnar D, Caputo ND, Chang BP, Chiricolo G, et al. Conscious proning: an introduction of a proning protocol for non-intubated, awake, hypoxic emergency department COVID-19 patients. Acad Emerg Med. 2020;27(suppl 7):566-69. https://doi.org/10.1111/acem.14035

3. Moghadam VD, Shafiee H, Ghorbani M, Heidarifar R. Prone positioning in management of COVID-19 hospitalized patients. Rev Bras Anestesiol. 2020;70(2):188-90. https://doi.org/10.1016/j.bjan.2020.05.001

4. Menezes, MR. Enfermagem gerontológica: um olhar diferenciado no cuidado biopsicossocial. São Paulo: Martinari; 2016.

5. Ghelichkhani P, Esmaeili M. Prone position in management of COVID-19 patients; a commentary. Arch Acad Emerg Med [Internet]. 2020[cited 2020 Oct 03];8(e48):1-3. Available from: https://www.ncbi.nlm.nih.gov/pmc/articles/PMC7158870/

6. Bamford P, Denmade C, Guidance for: prone positioning in adult critical care. J Intensive Care Soc Fac Intensive Care Med [Internet]. 2020[cited 2020 Oct 03]. Available from: https://www.ficm.ac.uk/sites/default/files/prone_position_in_adult_ critical_care_2019.pdf

7. Oliveira VM, Piekala DM, Deponti GN. Safe Prone checklist: construction and implementation of a tool for performing the prone maneuver. Rev Bras Ter Intensiva. 2017;29(suppl2):131-41. https://doi.org/10.5935/0103-507X.20170023

8. Borges DL, Rapello GVG, Deponti GND, Andrande FMDA. Posição prona no tratamento da insuficiência respiratória aguda na COVID-19. Rev ASSOBRAFIR Ciênc. 2020[cited 2020 Oct 03];11(suppl 1):1-276.2020. https://doi.org/10.47066/2177-9333. covid19.011

9. Marques Filho E, Maciel EP, Trindade RPA, Badaró BA, Cunha MP, Pereira MF. Manuseio do paciente com COVID-19 em unidade de terapia intensiva. Rev Cient HSI. 2020;4(suppl-2):105-23. https://doi.org/10.35753/rchsi.v4i2.177

10. Guirra PSB, Gomes JS, Biliu KS, Medved IV, Almeida VC. Manejo do paciente com Covid-19 em pronação e prevenção de lesão por pressão. Rev Health Resid J (HRJ). [Internet]. 2020;1 (suppl 2). Available from: https://escsresidencias.emnuvens. com.br/hrj/article/view/30/12 